\title{
SKA Deep Polarization and Cosmic Magnetism
}

\section{A.R. Taylor ${ }^{* a, b}$, Ivan Agudo ${ }^{c}$, Takuya Akahori ${ }^{d}$, Rainer Beck ${ }^{e}$, Bryan Gaensler ${ }^{f}$, George Heald ${ }^{g}$, Melanie Johnston-Hollitt ${ }^{h}$, Mathieu Langer ${ }^{i}$, Lawrence Rudnick ${ }^{j}$, Dongsu Ryu $^{k}$, Anna Scaife ${ }^{l}$, Dominik Schleicher ${ }^{m}$, and Jeroen Stil ${ }^{n}$}

${ }^{a}$ University of Cape Town, E-mail: russ a ast . uct . ac . ca

${ }^{b}$ University of the Western Cape, E-mail: russeast.uct.ac. ca

${ }^{c}$ Joint Institute for VLBI in Europe, E-mail: agudo@ jive.n 1

${ }^{d}$ University of Sydney, E-mail: akahoriaphysics . usyd.edu . au

${ }^{e}$ Max-Planck-Institut für Radioastronomy, E-mail: rbeck@mpifr-bonn .mpg . de

${ }^{f}$ University of Sydney, E-mail: bryan.gaens leresydney • edu . au

${ }^{g}$ ASTRON, E-mail: healdaastron.nl

${ }^{h}$ Victoria University of Wellington, E-mail: Melanie. Johnstron-Hollittevuw • ac.nz

${ }^{i}$ Université Paris-Sud, E-mail: mathieu. langer@ias.u-psud. fr

${ }^{j}$ University of Minnesota E-mail: larry@unm. edu

${ }^{k}$ UNIST, E-mail: ryuesirius.unist.ac.kr

${ }^{l}$ University of Southampton, E-mail: A. scaifedsoton.ac.uk

${ }^{m}$ Institut für Astrophysik Göttingen, E-mail:

dschleicaastro.physik.uni-goettingen.de

${ }^{n}$ University of Calgary, E-mail: jstileucalgary . ca

Deep surveys with the SKA1-MID array offer for the first time the opportunity to systematically explore the polarization properties of the microJy source population. Our knowledge of the polarized sky approaching these levels is still very limited. In total intensity the population will be dominated by star-forming and normal galaxies to intermediate redshifts $(z \sim 1-2)$, and low-luminosity AGN to high redshift. The polarized emission from these objects is a powerful probe of their intrinsic magnetic fields and of their magnetic environments. For redshift of order 1 and above the broad bandwidth of the mid-bands span the Faraday thick and thin regimes allowing study of the intrinsic polarization properties of these objects as well as depolarization from embedded and foreground plasmas. The deep field polarization images will provide Rotation Measures data with very high solid angle density allowing a sensitive statistical analysis of the angular variation of RM on critical arc-minute scales from a magnetic component of Large Scale Structure of the Universe.

Advancing Astrophysics with the Square Kilometre Array

June 8-13, 2014

Giardini Naxos, Italy

\footnotetext{
* Speaker.
} 


\section{Introduction}

The implementation of broad bandwidths and new correlator capacities on the Jansky Very Large Array (JVLA) and the Giant Meterwave Radio Telescope (GMRT) are opening up polarization imaging of the radio sky at mid-frequencies to $\mu$ Jy sensitivities (Rudnick \& Owen 2014, Taylor et al. 2014). However, the survey speeds of these instruments limit the amount of sky that can be imaged at these sensitivities in a reasonable time to a square degree or less, thus current studies of the polarized radio source population at these flux densities suffer from low number statistics. The MeerKAT SKA precursor continuum surveys will extend imaging at these sensitivities to larger areas (35 sq deg. to rms of $1.5 \mu \mathrm{Jy}$ ), albeit with lower angular resolution. At $75 \mathrm{nJy}$ rms and arc second resolution SKA1-MID can take a step in sensitivity of over an order magnitude compared to the present state-of-the-art and planned precursor projects, and will survey a large enough area of sky to provide a statistical overview of the magnetic properties of source populations over cosmic time.

Current studies hint at what the SKA1 deep polarized sky will reveal. Figure 1 shows at left a compilation of cumulative number counts of polarised sources at $1.4 \mathrm{GHz}$ down to $15 \mu \mathrm{Jy}$ from recent deep imaging projects. The plot demonstrates significant variation of results from different observers, likely due to both low number statistics and sensitivity to angular resolution. A slight flattening of the cumulative counts is suggested (Rudnick \& Owen 2014). This may reflect either a smaller overall number of polarized sources, perhaps arising from a decreasing fraction of Active Galactic Nuclei (AGN), and/or an on-average smaller fractional polarization for fainter sources. The latter is consistent with integrated polarization properties of disk galaxies in which internal depolarization effects from thermal plasma decreases the fractional polarization at frequencies below a few GHz (Stil et al. 2009, Heald et al. 2015). Faint polarized counts are thus expected to be frequency dependent and broad-band data are required to unravel the underlying astrophysics. The right hand of Figure 1 shows polarized source counts at $5 \mathrm{GHz}$ down to $5 \mu \mathrm{Jy}$ (Taylor et al. 2014). The rising blue dashed line shows the predicted polarized counts for disk galaxies based on the polarization properties of nearby galaxies (Stil et al. 2009). Deep imaging with SKA1 at GHz frequencies will probe the polarized properties of galaxies to high redshift.

\section{Emergence and Evolution of Magnetic Fields in Galaxies}

Through its ability to detect polarization of sources at high redshift, a deep survey is the cornerstone for investigation of the evolution of cosmic magnetic fields as it allows us to compare similar objects over the largest possible range in redshift, and place observations of local samples (e.g. Beck et al. 2015) in an evolutionary context. For disk galaxies, this range is $\mathrm{z} \gtrsim 2.5$, and for AGN and starbursts it reaches out to $\mathrm{z} \gtrsim 7$, into the epoch of reionization. Over this period, galaxies formed and evolved, converting most of their gas into stars. The evolution of plasma and magnetic fields in galaxies is therefore closely related to the evolution of the cosmic star formation rate, and tied to the intergalactic medium through accretion, galactic winds, tidal and ram stripping, and AGN activity.

With the SKA1 we can study magnetic fields over cosmic time through polarization of synchrotron emission in the source, Faraday rotation and depolarization, and Zeeman splitting. These 
typically require much higher sensitivity than detection of the source in total intensity. In the case of Faraday rotation and Zeeman splitting, chance alignment with a source in the background will allow detection of magnetic fields in sources that cannot be detected by their own emission. Since the background source must be at higher redshift, a deep field is more suitable to detect polarized background sources for high-redshift objects.

A majority of radio sources fainter than $100 \mu \mathrm{Jy}$ will be star-forming galaxies. The most luminous of these will be Ultra-luminous Infrared Galaxies (ULIRGs) and galaxy mergers. More common are relatively quiescent disk galaxies with a star formation rate that declines gradually by an order of magnitude from redshift 2 to the present. Deep surveys at other wavelengths are important to classify objects, and to measure the plasma density necessary to derive the magnetic field strength from Faraday rotation. In turn, a deep radio survey is required to detect polarization in high-redshift galaxies detected in infrared and optical surveys.

For star forming galaxies, evolution of the magnetic field is closely related to evolution of the galaxy itself. While $\mu \mathrm{G}$ strength magnetic fields with kpc scales should have formed in galaxy disks by $\mathrm{z}=3$, field ordering on the scale of a galaxy may have taken until $\mathrm{z}=0.5$, depending on galaxy mass (Arshakian et al. 2009). Competing with this, galaxy interactions and continuous feedback by supernovae and stellar winds that enhance the turbulent component of the magnetic field, and may drive outflows that transport plasma and magnetic field from the disk into the halo. Since these processes scale with the global star formation rate, significant evolution is expected between $\mathrm{z}=2$ and the present. Also, the density of Faraday rotating plasma will gradually decrease over time as galaxies transform a significant fraction of their gaseous mass into stars, implying a gradual evolution in Faraday depth. Figure 2 shows the predicted redshift distribution of galaxies with polarized flux density more than $10 \sigma$ in an SKA1-MID band $2 \& 3$ survey with sensitivity 75 nJy. These simulations are based on models by Stil et al. (2009) for spiral galaxies at redshift
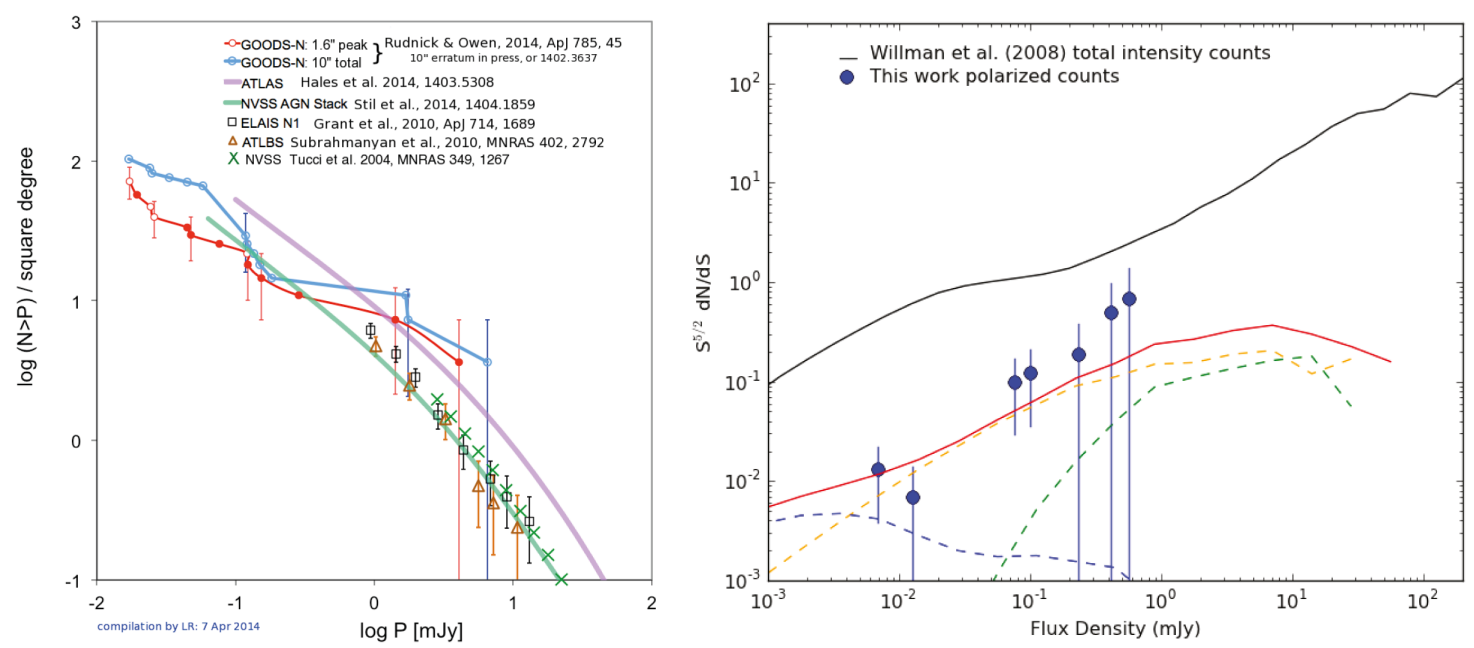

Figure 1: Left: Compilation of cumulative polarized source counts down at $1.4 \mathrm{GHz}$ to $\sim 15 \mu \mathrm{Jy}$ from recent studies. Right: Differential polarized source counts at $5 \mathrm{GHz}$ down to $5 \mu \mathrm{Jy}$ compared to predicted polarized counts of AGN (green and orange dashed lines) and disk galaxies (blue dashed line) [?]. 

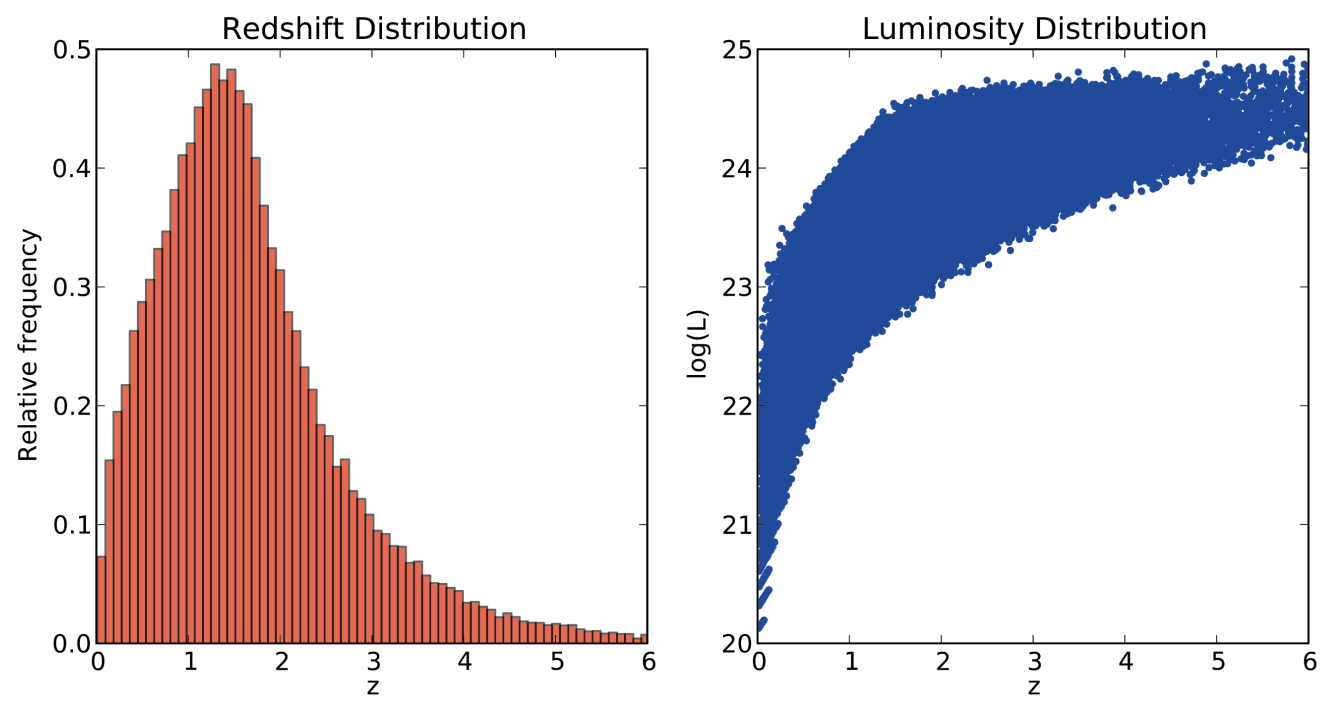

Figure 2: Left: Redshift distribution of normal star-forming galaxies with polarization properties of local disk galaxies that will have polarized flux density greater than $10 \sigma$ in the proposed SKA1 deep polarization field. Right: redshift versus radio luminosity of the same galaxies, showing that the galaxies probed are in the bright tail of the radio luminosity function and have significant luminosity evolution.

0, applied to normal star forming galaxies in the SKADS S3 simulation of Wilman et al. (2008). We expect to detect $\sim 5000$ galaxies per square degree above $10 \sigma$. A 10 square degree survey would thus provide probes of the presence of ordered magnetic fields in of order 50,000 galaxies out to redshift $>4$. The luminosity- redshift diagram for these galaxies shows that many will have experienced significant luminosity evolution to redshift 0. SKA1-MID is well-positioned to distinguish polarization properties of the more luminous regular star forming galaxies from those of their local (present day) counterparts. Zeeman splitting of HI absorption and Faraday rotation by systems in the line of sight to distant AGN will allow us to investigate magnetic fields in galaxies with radio emission below the detection limit of this survey.

Extreme starbursts in ULIRGS are environments that are very rare in the local universe. Little is known about magnetic fields in these objects. The dense interstellar medium suggests that any polarized emission related to star formation or an embedded AGN will be subject to very strong Faraday rotation. These objects may also have extended synchrotron halos associated with a galactic wind, or extended magnetic fields associated with tidal tails. These magnetic fields exist at the boundary of galactic and intergalactic space and may serve as seed fields for a more wide-spread intergalactic magnetic field.

\section{Magnetic Properties of AGN over Cosmic Time}

Active galactic nuclei are powered by supermassive black holes that accrete matter and are capable of forming pairs of relativistic jets that can extend up to distances far larger than the size of the host galaxy. The fact that jets in AGN are formed from magnetic systems, and that the magnetic field participating in their formation is partially dragged all along the jets, make polariza- 
tion observations of their synchrotron emission a powerful tool to test magneto-hydrodynamic and emission models. However, polarization studies of the radio AGN phenomenon have been limited by the lack of polarization purity, sensitivity, bandwidth and spectral resolution to make adequate studies of magnetic fields in AGN jets and their environments for massive samples of sources. A deep full-polarimetric SKA1-MID survey on Bands 2 and 3 reaching a sensitivity of $75 \mathrm{nJy} / \mathrm{beam}$ will have the ability to attack the long standing problem of the composition of AGN jets (i.e. the electron-proton content), and their plasma acceleration from large samples suited for statistical studies for the first time, see Agudo et al. (2015). For that, it is essential to have available a circular polarization purity at least $\sim 0.01 \%$, since AGN typically show circular polarization moduli in the range $0.1-1 \%$ at centimetre radio wavelengths.

The first large scale investigations on the polarization of AGN sources have been based on the NRAO VLA Sky Survey (NVSS) (Condon et al. 1998), which has detected 1.8 million extragalactic sources in total intensity. From these, $14 \%$ show a clear polarization signal of at least $3 \sigma$, and the majority of polarized extragalactic sources in the NVSS are AGN. Bright sources above $100 \mathrm{mJy}$ correspond to the radio-loud FRII sources (Fanaroff \& Riley 1974), and a gradual transition towards FRI sources occurs around $30 \mathrm{mJy}$. Around a flux density of $1 \mathrm{mJy}$, the contribution from starforming galaxies becomes more relevant. So far, typical deep imaging projects have been restricted to flux density sensitivities of $\sim 10 \mu \mathrm{J}$ in total intensity (Hopkins et al. 2003). While no dependence of polarization properties on redshifts out to $z \sim 3$ has been found, it is very interesting that surveys, including the NVSS, and the Dominion Radio Astrophysical Observatory Deep ELAIS N1 Field indicate an increase in fractional polarization with decreasing flux density (Mesa et al. 2002, Taylor et al. 2007). This anti-correlation suggests either a) a change in the magnetic field structure of the observed sources or b) a change of the properties their Faraday screen, perhaps related to the close intergalactic environment of the sources. Different solutions for this anti-correlation have been suggested, reflecting either a change in the population (Mesa et al. 2002), a change in the fraction of radio-quiet AGN or the FRII-FRI transition. From detailed studies of samples with high polarization, no strong dependence on optical morphology, redshift, linear size or radio power has been found (Shi et al. 2010), while a stacking analysis confirmed that the observed correlation continues toward very low flux densities in the NVSS (Stil et al. 2014). SKA1-MID will provide the means to take a major next step in this research, i.e. a deep polarimetric survey of the AGN populations up to the highest achievable sensitivities.

The superb sensitivity of SKA1 opens a new window to study the AGN phenomenon and its surrounding medium, from a cosmological perspective, back to the epoch of reionization, including the potential appearance of radio galaxies near or above $z \sim 10$, at a time where the densities in the Universe were considerably enhanced by a factor of $\sim 10^{3}$. Due to the enhanced densities in the interstellar and intergalactic medium, it becomes more difficult for jets to break out from their host galaxy (Falcke et al. 2004). Such systems known as GHz-Peaked-Spectrum (GPS) sources have been observed in the local Universe, corresponding to environments that are either very dense or where the jet is very young (ODea 1998). Due to the characteristic evolution of angular scales and observed frequency with redshift, the population of high-z GPS sources will likely be shifted towards lower frequencies, but will show sizes compared to those of their local counterparts (Falcke et al. 2004). The latter peak their radio spectrum at $\sim 1 \mathrm{GHz}$, hence making a deep SKA1-MID total intensity survey between 1 and $3 \mathrm{GHz}$ less suited for searches of high-z GPS 
than a parallel SKA1-LOW deep survey. In contrast, a deep and wide band survey at frequencies $>1 \mathrm{GHz}$, where the sources are expected to be a factor of $\sim 10$ fainter than in the SKA1-LOW bands, avoids strong Faraday depolarization by the dense environments in which the first AGN are expected to be embedded, and therefore perhaps allowing the detection of their linear polarization. A deep-polarimetric and wide-band SKA1-MID survey in Bands 2 and 3 hence opens the exciting possibility not only to probe the magnetic fields in the first AGN jets, but also their immediate dense intergalactic medium at $z \sim 10$.

\section{Detecting Magnetic Fields in the Cosmic Web}

The SKA1 deep polarization survey will provide the supremely-dense RM grid of polarization probes of the intergalactic medium required to detect and measure the properties of magnetic fields embedded in the large scale structure of the universe. The LCDM cosmology predicts the cosmic web of galaxy clusters and filaments. While clusters contain hot plasma of $\mathrm{T}>10^{7} \mathrm{~K}$, filaments are filled with plasma of $10^{7} \mathrm{~K}>\mathrm{T}>10^{5} \mathrm{~K}$ which is referred to as the Warm Hot Intergalactic Medium (WHIM). The plasmas are expected to be magnetized; diverse processes for seed magnetic fields have been suggested, and the seed fields can be further amplified through compression and turbulent dynamo as well as leakage of galactic media during the hierarchical structure formation in the Universe. See Ryu et al. (2012) and Widraw et al. (2012) for reviews.

The Intergalactic Magnetic Field (IGMF) in the cosmic web plays important roles in various astrophysical phenomena. For instance, the IGMF imprints its own existence on the cosmic microwave background, deflects the trajectory of cosmic-rays through the cosmic web, and it affects the thermal and dynamical evolutions of galaxy clusters. In addition, the IGMF may have provided seed fields of galaxies and influenced the origin and nature of magnetic fields in spiral galaxies.

RM has been the main tool for studies of the IGMF. It has revealed $B \sim 1-0 \mu \mathrm{G}$ in clusters. Detection of magnetic fields in filaments is challenged due to the expected very low value of RM. Cosmological simulations suggest the IGMF in filaments may have $B \sim 1-100 \mathrm{nG}$ (Ryu et al. 2008). With the IGMF, the rms value of $\mathrm{RM}, \mathrm{RM}_{\mathrm{rms}}$, through a single filament would be $\sim 1$ $\mathrm{rad} \mathrm{m}^{-2}$ (Figure 3 left, (Akahori \& Ryu 2010)), while $\mathrm{RM}_{\mathrm{rms}}$ through a number of filaments up to $\mathrm{z} \sim \mathrm{a}$ few would reach several $\mathrm{rad} \mathrm{m}^{-2}$ (Figure 3 middle, (Akahori \& Ryu 2011)). Interestingly, such RM is comparable to the estimate of extragalactic contribution to the observed RM of $6-15$ $\mathrm{rad} \mathrm{m}^{-2}$ (Schnitzeler 2010, Hammond et al. 2012).

Simulations predict that the IGMF in filaments would induce RM with a flat second-order structure function (SF) of $\sim 100 \mathrm{rad}^{2} \mathrm{~m}^{-4}$ for angular separation of $\mathrm{r} \gtrsim 0.1^{\circ}$ (Figure 3 right). Toward high galactic latitudes where the Galactic contribution is minimum, the Galactic magnetic field, on the other hand, should produce substantially smaller and steeper SF in angular scale (Akahori et al. 2013). Observed SFs toward high latitude, while poorly sampled, are consistent with a flat SF on the smallest scales (Mao et al. 2010, Stil et al. 2011), suggesting a contribution from the IGMF on scales below $1^{\circ}$.

Directions toward high Galactic latitude and toward outside of galaxy clusters should be chosen for deep-field surveys to detect the RM of the IGMF in cosmic filaments. The RM due to the Galactic magnetic field could be substantially reduced with spatial filtering to remove the large scale variance (Akahori et al. 2014). Simulations have shown that a RM data set with a sky den- 

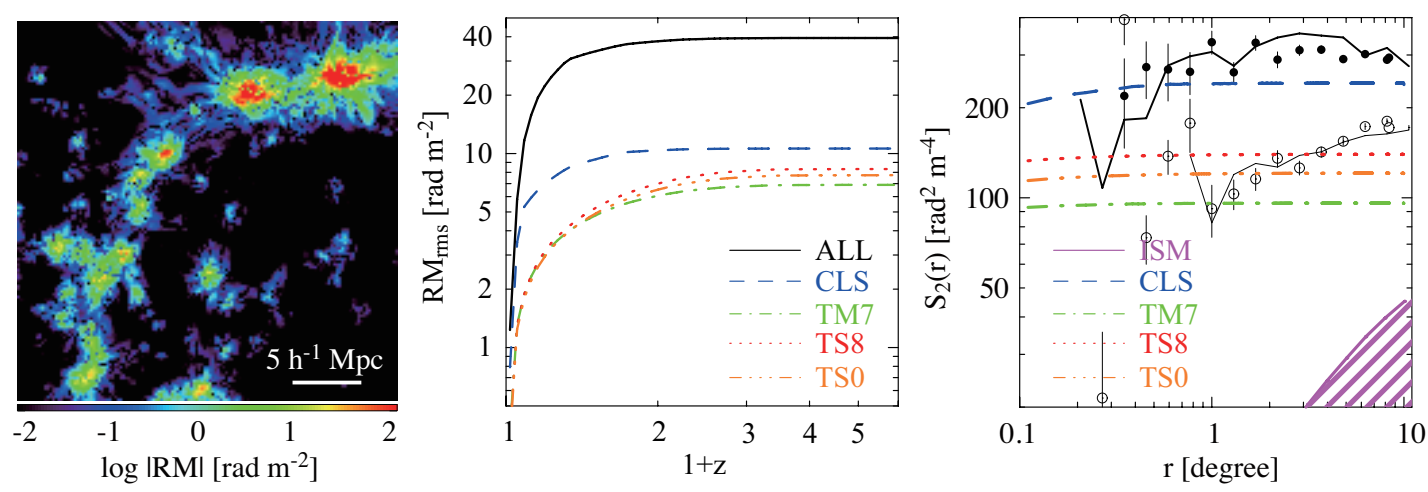

Figure 3: Left: The RM map of the local universe of a $100 h^{-1} \mathrm{Mpc}$ depth (Akahori \& Ryu 2010). Middle: The rms value of RM integrated up to $z=5$ (Akahori \& Ryu 2011). Results with different methods for cluster subtractions are shown as different colors. Right: Second-order structure functions of RM. Black circles (Mao et al. 2010) and lines (Stil. et al. 2011) are observed ones for $\sim 900 \mathrm{deg}^{2}$ fields-of-view toward North (filled, thick) and South (open, thin) Galactic poles. The purple area indicates possible amplitudes for the Milky Way RM toward the poles (Akahori et al. 2013).

sity of several 100 to 1000 per square degree and with RM precision of $\sim 1 \mathrm{rad} \mathrm{m}^{-2}$ is required to accurately reconstruction the structure function of RM variance due to the IGMF in the cosmic web (Akahori et al. 2014). An SKA1-MID deep polarization survey to below $100 \mathrm{nJy}$ with band 2 \& 3 would yield formal errors in RM of $1 \mathrm{rad} \mathrm{m}^{-2}$ for sources with polarized flux density greater than $1.3 \mu \mathrm{Jy}$. At this polarized flux density the source density will approach the required 1000 per square degree (see Figure 1). A significant fraction of this population will be galaxies. The alignment of polarization position angle with optical minor axis seen in local disk galaxies (Stil et al. 2009) offers the possibility to also use polarization and optical alignments of galaxies as another powerful probe of weak Faraday Rotation from large scale structure.

\section{Technical Summary}

The Cosmic Magnetism Deep Fields are intended to be observed commensally with the continuum survey deep fields using SKA1-MID over a frequency range of $950-3050 \mathrm{MHz}$ (Band $2 \& 3)$. Band 3 will sample the regime where internal Faraday depolarization effects are negligible and alignment of polarization and geometric properties of the galaxy are preserved. Band 2 samples the depolarization regime and allows measurement of internal Faraday processes from galaxy structure (Heald et al. 2015). The significant depolarization in band 2 for nearby galaxies will be substantially reduced at $\mathrm{z}>1$. Emission from galaxies in SKA1-MID Band 1 will suffer very significant depolarization for the red shifts available to SKA1. SKA1 Band 1 and 2 frequency coverage will provide formal precision better than RM of $1 \mathrm{rad} \mathrm{m}^{-2}$ down to polarized intensity of $1 \mu \mathrm{Jy} \mathrm{beam}^{-1}$. Integration times sufficient for a sensitivity of $75 \mathrm{nJy}$ beam $^{-1}$ are required per field at a resolution of 1 arcsec. At this resolution the continuum (monochromatic) rms noise for SKA1-MID is expected to be twice (three times) that of the naturally weighted visibility data for Band 2, given current array configuration projections.

Star-forming galaxies with partly ordered regular fields are expected to have Faraday depth of 
$\sim 50 \mathrm{rad} \mathrm{m}^{-2}$ at $z \sim 0.5$. Large-scale field reversals may exist in those epochs and lead to several Faraday Depth components with different signs, so that a resolution of $50 \mathrm{rad} \mathrm{m}^{-2}$ is required, Band $2 \& 3$ combine to provide Faraday depth resolution of $31 \mathrm{rad} \mathrm{m}^{-2}$

The majority of galaxies are expected of exhibit integrated polarized fraction of order 1 to a few percent, so linear polarization purity of $0.1 \%$ across the field of view is required. Circular polarization purity of $0.01 \%$ would allow circular polarization to be studied for the fist time in a vast number of AGN.

Imaging of an area of the order 10 square degrees is required to provide statistical sampling of magnetic fields in galaxies and AGN and for sampling of large-scale structure for detection of the cosmic magnetic web. We expect a several tens of thousands of objects detected in polarization for study of the evolution of intrinsic magnetic fields with cosmic time. The dense grid of thousands of sources per square degree will probe the "foreground" cosmic magnetic web.

Both continuum and spectral imaging will be required in full polarization for these data with a coarse spectral resolution of $\Delta v=1 \mathrm{MHz}$. Processing of the polarization deep fields will require some additions to a standard imaging pipeline. Channelized polarization products will need to be formed in full Stokes with high dynamic range per channel. To avoid contamination from bright sources in the fields a dynamic range requirement of $10^{6}\left(10^{5}\right)$ in total intensity (polarization) is required for continuum imaging and $10^{4}\left(10^{3}\right)$ per channel for spectral data. To achieve this dynamic range, full Stokes A-projection will be required in addition to standard W-projection. Direction-dependent calibration will be necessary, followed by multifrequency synthesis.

These fully calibrated polarization data can then be transformed from frequency into Faraday dispersion cubes. Standard RM-synthesis would utilize the channelized QU image cube (Brentjens $\&$ de Bruyn 2005). However to achieve full dynamic range in the Faraday dispersion cube images from the broad bandwidth will require multi-frequency synthesis techniques to be adapted to Stokes Q, U data. Integration of multi-frequency synthesis and Faraday dispersion imaging would require Faraday de-dispersion to operate on the visibilities prior to imaging and deconvolution.

\section{REFERENCES}

Agudo, I. et al. 2015, "Studies of Relativistic Jets in Active Galactic Nuclei with SKA" in proc. Advancing Astrophysics with the SKA, PoS(AASKA14)093

Akahori T., \& Ryu D. 2010, ApJ, 723, 476

Akahori T., \& Ryu D. 2011, ApJ, 738, 134

Akahori T., Ryu D., Kim J., \& Gaensler B. M. 2013, ApJ, 767, 150

Akahori T., Gaensler, B. M., \& Ryu, D., 2014, ApJ, 790. 123

Arshakian, T. G., Beck, R., Krause, M., \& Sokoloff, D. 2009, AA, 494, 21

Beck, R. et al. 2015, "Structure, dynamical impact and origin of magnetic fields in nearby galaxies in the SKA era" in proc. Advancing Astrophysics with the SKA, PoS(AASKA14)094

Brentjens, M.A. \& de Bruyn, A.G. 2005, A\&A, 441, 1217

Condon, J. J. et al. 1998, AJ, 115, 1693

Falcke, H. et al. 2004, NewAR, 48, 1157

Fanaroff, B.L. \& Riley, J.M., 1974, MNRAS, 1767, 31

Hammond, A. M., Robishaw, T., \& Gaensler, B. M. 2012 (arXiv:1209.1438v3) 
Heald, G. et al. 2015, "Magnetic Field Tomography in Nearby Galaxies with the Square Kilometre Array" in proc. Advancing Astrophysics with the SKA, PoS(AASKA14)093

Hopkins, A. M. et al. 2003, AJ, 125, 465

Mao, S. A., Gaensler, B. M., Haverkorn, M., et al. 2010, ApJ, 714, 1170

Mesa, D. 2002, A\&A, 396, 463

O'Dea, C. 1998, PASP, 110, 493

Rudnick, L. and Owen, F.N., 2014, ApJ, 785, 45

Ryu, D., Kang, H., Cho, J., \& Das, S. 2008, Science, 320, 909

Ryu, D., et al. 2012, Space Science Review, 166, 1

Schnitzeler, D. H. F. M. 2010, MNRAS, 409, 99

Shi, H. et al. 2010, MNRAS, 409., 821

Stil, J. M., Krause, M., Beck, R., \& Taylor, A. R. 2009, ApJ, 693, 1392

Stil, J. M., Taylor, A. R., \& Sunstrum, C. 2011, ApJ, 726, 4

Stil, J. M., Keller, B.W., George, S.J. and Taylor A.R. 2014, ApJ, 797, 99

Taylor, A. R., Stil, J.M., Grant, J.K. et al. 2007, ApJ, 666, 201

Taylor, A.R., Bhatnagar, S., Condon, J. et al., 2014, BASI, arXiv:1405.0117

Widraw, L., et al. 2012, Space Science Review, 166, 37

Wilman, R. J., Miller, L., Jarvis, M. J., Mauch, T., Levrier, F., Abdala, F. B., Rawlings, S., Klöckner, H.-R., Obreschkow, D., Olteanu, D. \& Young, S. 2008, MNRAS, 388, 1335 